\title{
Efficacy Results of a Trial of a Herpes Simplex Vaccine
}

\author{
Robert B. Belshe, M.D., Peter A. Leone, M.D., David I. Bernstein, M.D., Anna Wald, M.D., \\ Myron J. Levin, M.D., Jack T. Stapleton, M.D., Iris Gorfinkel, M.D., Rhoda L. Ashley Morrow, \\ Ph.D., Marian G. Ewell, Sc.D., Abbie Stokes-Riner, Ph.D., Gary Dubin, M.D., Thomas C. \\ Heineman, M.D., Ph.D., Joann M. Schulte, D.O., Carolyn D. Deal, Ph.D., and for the \\ Herpevac Trial for Women \\ Division of Infectious Diseases, Allergy, and Immunology, Saint Louis University, St. Louis \\ (R.B.B.); the Department of Epidemiology, University of North Carolina, Chapel Hill (P.A.L.); the \\ Division of Infectious Diseases, Cincinnati Children's Hospital Medical Center, Cincinnati (D.I.B.); \\ the Department of Medicine, Epidemiology, and Laboratory Medicine, Fred Hutchinson Cancer \\ Research Center, Vaccine and Infectious Diseases Division (A.W.), and Children's Hospital \\ Virology, Department of Laboratory Medicine (R.L.A.M.), University of Washington, Seattle; the \\ Anschutz Medical Campus, Section of Pediatric Infectious Diseases, University of Colorado \\ Denver, Aurora (M.J.L.); the Department of Internal Medicine, University of lowa, and the \\ Veterans Affairs Medical Center — both in lowa City (J.T.S.); the PrimeHealth Clinical Research \\ Organization, Toronto (I.G.); the EMMES Corporation, Rockville (M.G.E., A.S.-R.), and the \\ National Institute of Allergy and Infectious Diseases, Bethesda (J.M.S., C.D.D.) - both in \\ Maryland; and GlaxoSmithKline, King of Prussia, PA (G.D., T.C.H.).
}

\begin{abstract}
Background-Two previous studies of a herpes simplex virus type 2 (HSV-2) subunit vaccine containing glycoprotein D in HSV-discordant couples revealed $73 \%$ and $74 \%$ efficacy against genital disease in women who were negative for both HSV type 1 (HSV-1) and HSV-2 antibodies. Efficacy was not observed in men or HSV-1 seropositive women.

Methods-We conducted a randomized, double-blind efficacy field trial involving 8323 women 18 to 30 years of age who were negative for antibodies to HSV-1 and HSV-2. At months 0,1 , and 6 , some subjects received the investigational vaccine, consisting of $20 \mu \mathrm{g}$ of glycoprotein D from HSV-2 with alum and 3-O-deacylated monophosphoryl lipid A as an adjuvant; control subjects received the hepatitis A vaccine, at a dose of 720 enzyme-linked immunosorbent assay (ELISA) units. The primary end point was occurrence of genital herpes disease due to either HSV-1 or HSV-2 from month 2 (1 month after dose 2) through month 20.
\end{abstract}

Copyright $(2012$ Massachusetts Medical Society.

Address reprint requests to Dr. Belshe at the Saint Louis University School of Medicine, Division of Infectious Diseases, Allergy, and Immunology, 1100 S. Grand Blvd., DRC-8, St. Louis, MO 63104, or at belsherb@slu.edu..

Dr. Belshe reports serving as a board member of Vivaldi Biosciences, receiving consulting fees from GlaxoSmithKline, receiving consulting fees and lecture fees from MedImmune, and receiving lecture fees from Merck. Dr. Bernstein reports receiving lecture fees and royalties from GlaxoSmithKline. Dr. Dubin reports being an employee of and receiving stock and travel, accommodation, and meeting expenses from GlaxoSmithKline and receiving royalties from Pfizer. Dr. Gorfinkel reports receiving grants, consulting fees, travel expenses to meetings, fees for participating in review activities, equipment and technical assistance, lecture fees, and payment for developing educational presentations from GlaxoSmithKline, having stock equity in and serving as an investigator for GlaxoSmithKline, and serving as an investigator for AstraZeneca, Bristol-Myers Squibb, Janssen-Ortho, Bayer, PharmaNet, Wyeth, Berlex, Lundbeck, and CombinatoRx. Dr. Heineman reports being an employee of GlaxoSmithKline and receiving stock equity in GlaxoSmithKline as part of his compensation. Dr. Leone reports receiving consulting fees and speaker fees from GlaxoSmithKline, lecture fees from Novartis and Abbott Diagnostics, and grant support from Genocea. Dr. Levin reports receiving consulting fees and grants from Glaxo SmithKline. Dr. Morrow reports receiving consulting fees from Roche. Dr. Schulte reports owning stock in Pfizer. Dr. Stapleton reports receiving grant support from GlaxoSmithKline. Dr. Wald reports receiving consulting fees from AiCuris and grant support from the Washington Vaccine Alliance. No other potential conflict of interest relevant to this article was reported. Disclosure forms provided by the authors are available with the full text of this article at NEJM.org. 
Results-The HSV vaccine was associated with an increased risk of local reactions as compared with the control vaccine, and it elicited ELISA and neutralizing antibodies to HSV-2. Overall, the vaccine was not efficacious; vaccine efficacy was $20 \%$ (95\% confidence interval [CI], -29 to 50) against genital herpes disease. However, efficacy against HSV-1 genital disease was 58\% (95\% CI, 12 to 80). Vaccine efficacy against HSV-1 infection (with or without disease) was 35\% (95\% CI, 13 to 52 ), but efficacy against HSV-2 infection was not observed (-8\%; 95\% CI, -59 to 26 ).

Conclusions-In a study population that was representative of the general population of HSV-1- and HSV-2-seronegative women, the investigational vaccine was effective in preventing HSV-1 genital disease and infection but not in preventing HSV-2 disease or infection. (Funded by the National Institute of Allergy and Infectious Diseases and GlaxoSmithKline; ClinicalTrials.gov number, NCT00057330.)

Both herpes simplex virus type 1 (HSV-1) and type 2 (HSV-2) can cause primary infection of the genital tract, and HSV-1 infection has become an increasingly frequent cause of genital disease. ${ }^{1}$ The majority of HSV infections are asymptomatic, and only 10 to $25 \%$ of persons with HSV-2 antibodies have recurrent genital disease. ${ }^{2,3}$ Transmission of HSV from infected women to neonates may lead to severe neurologic disease or death in the newborn. Strategies to control genital herpes infection and disease have mainly focused on antiviral chemotherapy, education, and the use of condoms. The availability of an effective prophylactic vaccine would help control genital herpes.

In two previous efficacy trials of an HSV-2 glycoprotein D-based subunit (gD-2) vaccine in discordant couples in which one partner had recurrent HSV genital disease, the subset of seronegative women (negative for both HSV-1 and HSV-2 antibodies) was significantly protected against HSV-2 disease by the vaccine (73\% and $74 \%$ efficacy, respectively); efficacy was not shown in either men or HSV-1-seropositive women. ${ }^{4}$ To further evaluate the $\mathrm{gD}-2$ vaccine as a potential public health tool, we evaluated this vaccine in a cohort of women who were screened and found to be antibody-negative for HSV-1 and HSV-2.

\section{Methods}

\section{Study Population}

Women 18 to 30 years of age who were sero-negative for HSV-1 and HSV-2 were recruited from 40 sites in the United States and 10 in Canada. Other inclusion criteria were written informed consent, the absence of serious health problems, a willingness to use effective methods of birth control throughout the period from 30 days before vacci-nation to 2 months after receipt of the third dose of vaccine, and a negative pregnancy test.

\section{Vaccines and Adjuvants}

The HSV-2 vaccine (GlaxoSmithKline) contained $20 \mu \mathrm{g}$ of truncated glycoprotein D from HSV-2 strain G. The gD-2 vaccine antigen was combined with an adjuvant that consisted of $0.5 \mathrm{mg}$ of aluminum hydroxide (alum) and $50 \mu \mathrm{g}$ of 3-O-deacylated monophosphoryl lipid A. The vaccine was administered by injection at a dose of $0.5 \mathrm{ml}$ at 0,1 , and 6 months.

The control vaccine, inactivated hepatitis A vaccine (Havrix, GlaxoSmithKline), was formulated as 720 enzyme-linked immunosorbent assay (ELISA) units of inactivated hepatitis A virus combined with $0.5 \mathrm{mg}$ of alum, in a volume of $0.5 \mathrm{ml}$. For the study to be blinded, the control vaccine was given at 0,1 , and 6 months in doses containing one half the usual volume and one half the usual amount of antigen. 


\section{Study Design}

The double-blind, randomized field trial was designed in collaboration among the trial sponsors, the National Institutes of Health (NIH) and Glaxo-SmithKline; the study chair; the executive committee; and the scientific leadership group. Data were collected with the use of the GlaxoSmith-Kline remote data-entry system and were monitored by GlaxoSmithKline. All the authors and the trial sponsors vouch for the accuracy and completeness of the data. Data were electronically transferred to EMMES, a contract research organization, where they were analyzed according to the analysis plan prepared by biostatisticians at GlaxoSmithKline and EMMES, with input to the analysis plan from the $\mathrm{NIH}$, the study chair, and the executive committee. The manuscript was drafted by the first author, with input from the biostatisticians at EMMES and from the executive committee. All authors vouch for the fidelity of this report to the protocol, which is available with the full text of this article at NEJM.org.

After written informed consent had been obtained, subjects were randomly assigned in a 1:1 ratio (Fig. 1) to receive either the HSV vaccine or the control (hepatitis A) vaccine by intramuscular injection in the deltoid area at months 0,1 , and 6 . Active surveillance for suspected intercurrent HSV disease was conducted monthly by telephone, e-mail, text messaging, or social-network website, depending on the method selected by each subject. Serum specimens for surveillance of asymptomatic infection were obtained at $0,2,6,7,12$, 16, and 20 months. Subjects filled out a questionnaire at baseline and at each serologic time point to assess sexual-risk behavior. If genital or nongenital herpes disease was suspected at any time, the subject was examined, viral cultures were obtained, and treatment was initiated with valacyclovir at the discretion of the local investigator.

\section{Study End Points}

The primary end point of the study was prevention of genital herpes disease caused by HSV-1, HSV-2, or both from month 2 (1 month after vaccine dose 2) through month 20. Genital disease was defined as clinically compatible signs and symptoms confirmed by viral culture, seroconversion, or both within 6 months after disease onset. Secondary end points included prevention of HSV-1 or HSV-2 infection (with or without disease) from month 2 through month 20 (two-dose efficacy) or month 7 through month 20 (three-dose efficacy) and prevention of genital herpes disease caused by individual HSV types. Cases of infection and disease were determined centrally by an independent, blinded end-point review committee with the use of documented criteria.

\section{Substudy of Viral Shedding}

Subjects identified as having acquired genital HSV-2 disease or HSV-2 infection during the study were invited to participate in an evaluation of viral shedding. Subjects were instructed to collect daily swabs from the anogenital area for 60 consecutive days and to maintain a diary of genital signs and symptoms, as previously described, beginning 3 to 6 months after HSV-2 seroconversion or disease onset. ${ }^{5}$

\section{Laboratory Studies}

Western blot analysis (University of Washington Clinical Virology Laboratory at Seattle Children's Hospital) was used to confirm HSV-1- or HSV-2- seronegative status at study entry and seroconversion during the follow-up period. ${ }^{6}$ Seroconversion to HSV-1 or HSV-2 was defined as a positive Western blot analysis in a subject with a previously negative analysis for the corresponding HSV type (for details, see the Supplementary Appendix, available at NEJM.org). 
Serum specimens from a random subset of 611 subjects in the HSV-vaccine group and 223 subjects in the control group were assessed for the development of antibodies to vaccine antigens with the use of ELISA ${ }^{4,7}$ for gD-2 and virus neutralization ${ }^{8,9}$ of HSV-2 at 0, 2, 6, 7, 12,16 , and 20 months. Long-term genital shedding of HSV-2 DNA was assessed with the use of a quantitative, real-time, fluorescence-based polymerase-chain-reaction assay, as described previously, with a positive result defined as 150 copies per milliliter. ${ }^{10}$

\section{Statistical Analysis}

The trial was designed to have $80 \%$ power to detect a vaccine efficacy of $75 \%$ with $45 \%$ as the lower limit of the $95 \%$ confidence interval. It met the information goal, observing 70 of a planned 72 cases of genital herpes disease in the per-protocol cohort. The trial was monitored by an independent data safety and monitoring board sponsored by the National Institute of Allergy and Infectious Diseases, which met quarterly and reviewed the study for safety. At a prespecified interim analysis, the board also reviewed the trial for futility. The sample size was extended once in response to higher-than-anticipated attrition. Vaccine efficacy was estimated as 1 minus the relative risk from a Cox proportional-hazards model fit to the time to first acquisition of each study end point. Rates of loss-to-follow-up were similar between the two study groups, and noninformative censoring was assumed. A post hoc assessment of demographic and behavioral risk factors for HSV acquisition was performed with the use of a Cox proportional-hazards model adjusted for the receipt of HSV vaccine. All reported $P$ values are two-tailed and have not been adjusted for multiple testing. The per-protocol and intention-to-treat cohorts are defined in the legend for Figure 1.

\section{Results}

\section{Characteristics of the Study Population}

Fifty clinical sites in the United States and Canada screened a total of 31,770 women for antibodies to HSV-1 and HSV-2; 12,468 women were seronegative for both HSV-1 and HSV-2, of whom 8323 met the other eligibility criteria and were enrolled between January 14, 2003, and November 19, 2007. The demographic characteristics and self-reported baseline risk behaviors of the subjects in the per-protocol population (the study population for evaluation of the primary end point) are summarized in Table 1 . The two study groups were well balanced with respect to demographic characteristics and risk behaviors at study entry.

\section{Vaccine Efficacy}

The HSV vaccine was not efficacious with regard to the primary end point, protection against genital disease caused by either HSV-1 or HSV-2 after two doses of vaccine (Table 2 and Fig. 2A); overall vaccine efficacy was $20 \%$ (95\% confidence interval [CI], -29 to 50). In the control group, HSV-1 was a more common cause of genital disease than HSV-2 (21 cases caused by HSV-1 vs. 14 cases caused by HSV-2). Efficacy against genital disease caused by HSV-1 was observed (vaccine efficacy, 58\%; 95\% CI, 12 to 80) (Fig. 2B), but efficacy was not observed against HSV-2 disease ( $-38 \%$; 95\% CI, -167 to 29) (Fig. 2C). Three doses of vaccine were associated with efficacy against HSV-1 (77\%; 95\% CI, 31 to 92) but not HSV-2 (-40\%; 95\% CI, -234 to 41). An analysis in which the case definition was limited to culture-positive cases (excluding HSV cases diagnosed according to clinical and serologic criteria) also showed efficacy against HSV-1 (two-dose efficacy, 69\%; 95\% CI, 25 to 87; three-dose efficacy, 82\%; 95\% CI, 35 to 95).

The HSV vaccine provided protection against infection caused by HSV-1 or HSV-2 (efficacy, 22\%; 95\% CI, 2 to 38) (Table 2, and the Supplementary Appendix). This overall finding of protection against infection was driven by efficacy against HSV-1 infection (35\%; 
95\% CI, 13 to 52), whereas efficacy against HSV-2 infection was not observed (-8\%; $95 \%$ $\mathrm{CI},-59$ to 26$)$.

\section{Risk Factors for HSV Acquisition}

Self-reported behaviors and demographic factors were analyzed for association with HSV-1 or HSV-2 infection in the per-protocol cohort for months 2 through 20. Analysis of selfreported behavioral risk factors was restricted to 5980 sexually active subjects. An increased risk of HSV-1 infection was associated with 6 or more lifetime sexual partners (hazard ratio, 2.2; $95 \% \mathrm{CI}, 1.3$ to 3.8 ) and more than 1 partner in the previous 12 months (hazard ratio, $1.9 ; 95 \%$ CI, 1.4 to 2.7$)$. Subjects who were 23 years of age or older were less likely to acquire HSV-1 than 18-to-22-year-olds (hazard ratio for subjects 23 to 26 years of age, 0.6; $95 \%$ CI, 0.4 to 0.8 ; hazard ratio for subjects 27 to 30 years of age, $0.4 ; 95 \% \mathrm{CI}, 0.3$ to 0.8 ). Factors not associated with an increased risk of HSV-1 infection included race or ethnic group, country of residence (United States or Canada), having a current partner with herpes, ever having a partner with herpes, condom use, history of any sexually transmitted infection (STI), and oral sex.

An increased risk of HSV-2 infection was associated with having 6 or more lifetime sexual partners (hazard ratio, 2.0; 95\% CI, 1.1 to 3.8), having 6 or more partners in the previous 12 months (hazard ratio, $2.7 ; 95 \% \mathrm{CI}, 1.3$ to 5.5 ), ever having a partner with herpes (hazard ratio, 3.0; 95\% CI, 1.7 to 5.3), having a current partner with herpes (hazard ratio, 3.4; 95\% CI, 1.8 to 6.4 ), a history of any STI (hazard ratio, 3.3; 95\% CI, 2.2 to 5.0 ), nonwhite race (hazard ratio, 3.1; 95\% CI, 2.1 to 4.6), and U.S. residence (hazard ratio, 2.7; 95\% CI, 1.2 to 6.2). Factors not associated with increased risk of HSV-2 infection included age, ethnic group, condom use, and oral sex. Initiation of sexual activity after 15 years of age was associated with a decreased risk of both HSV-1 infection (hazard ratio for initiation at 16 to 18 years of age, $0.6 ; 95 \% \mathrm{CI}, 0.4$ to 0.8 ; hazard ratio after 18 years of age, $0.3 ; 95 \% \mathrm{CI}, 0.2$ to 0.6 ) and HSV-2 infection (hazard ratio for initiation at 16 to 18 years of age, $0.5 ; 95 \% \mathrm{CI}$, 0.3 to 0.8 ; hazard ratio after 18 years of age, $0.3 ; 95 \% \mathrm{CI}, 0.2$ to 0.6 ).

\section{Genital Shedding of HSV-2}

Forty-three subjects (30 in the HSV-vaccine group and 13 in the control group) with HSV-2 infection collected anogenital swabs on 60 consecutive days, beginning 3 to 6 months after disease onset (15 subjects in the HSV-vaccine group and 9 in the control group) or seroconversion (15 subjects in the HSV-vaccine group and 4 in the control group) (Fig. 5 in the Supplementary Appendix). Analysis of these swabs showed that the rate of viral shedding was higher among the HSV-vaccine recipients than among controls (29\% vs. 19\%; relative risk, $1.55 ; 95 \% \mathrm{CI}, 1.28$ to 1.86 ). The mean quantity of HSV DNA on days with shedding did not differ between the two groups.

\section{Vaccine Safety}

Solicited reports of adverse events included redness, swelling, and pain at the injection site, as well as fatigue, fever, headache, and malaise (Table 3). The HSV vaccine was more reactogenic and was associated with local pain, redness, and swelling more often than was the control vaccine. There was a small but significant increase in systemic symptoms, including fatigue, fever, headache, and malaise, in the HSV-vaccine group (Table 3). Dose 2 and dose 3 of the HSV vaccine were not associated with increased reports of adverse events; in contrast, reactogenicity decreased slightly with additional vaccination. 


\section{Immunogenicity}

The HSV vaccine was immunogenic and stimulated ELISA and neutralizing antibodies. As expected, control subjects did not have antibody to gD-2 on ELISA or neutralization of HSV-2. Geometric mean gD-2 ELISA titers were 21 at baseline and 6809 at month 7 after three doses of HSV vaccine; ELISA titers waned to 769 by month 20 (see the Supplementary Appendix for details). HSV-2 neutralizing antibodies developed after two doses of HSV vaccine, but the median value fell to an undetectable level by study month 6 (see the Supplementary Appendix for details). After dose 3, HSV-2 neutralizing antibodies were again above the limit of detection (the mean titer at month 7 was 29) but fell to a median value that was undetectable by study month 16 .

\section{Discussion}

Our findings of vaccine efficacy against HSV-1 and lack of efficacy against HSV-2 are puzzling in view of the previous two studies involving discordant couples that showed efficacy of this gD-2 vaccine against HSV-2. ${ }^{4}$ The difference in efficacy is likely to be due to some factor in the two populations studied. The distinguishing feature of discordant couples is that they are a highly selected group in which the uninfected partner is potentially repeatedly exposed to HSV by the infected partner. Attack rates of HSV-2 genital disease in the prior studies of gD-2 vaccine were high among uninfected women in discordant couples (13.9\% for 19 months or $8.4 \%$ per year) and were reduced significantly by the vaccine (efficacy, $73 \%$ and $74 \%$ in the two trials; $\mathrm{P}=0.01$ and $\mathrm{P}=0.02$, respectively). ${ }^{4}$ Too few cases of HSV-1 genital disease occurred in women in the two previous studies to assess efficacy against HSV-1 (one case in each study among women who were sero-negative at study entry). ${ }^{4}$ Potential reasons for vaccine efficacy in the discordant-couple population include selection bias for a population of women with relative resistance to HSV-2, with added benefits from a subunit vaccine; an undefined immunologic priming event from chronic sexual exposure to HSV-2 viral antigens from the infected partner; and less frequent sexual activity due to the long-term nature of the relationship as compared with sexual activity by couples in new relationships.

It is not apparent why the biologic characteristics of HSV-1 are different from those of HSV-2; the gD-2 vaccine induces significant protection against genital HSV-1 disease as well as HSV-1 infection, but not against disease or infection caused by HSV-2. Genital HSV-1 may be acquired primarily through oral-genital sex (although a history of oral sex was not a risk factor for HSV-1 acquisition in our study); a lower inoculum, an oral- genital route (possibly less traumatic sex), and a less suitable environment for HSV-1 replication are all possible explanations for protection by the vaccine against HSV-1 but not HSV-2. The gD-2 antigen is derived from HSV-2, but $89 \%$ amino acid homology is shared with gD-2 from HSV-1, which may explain the protection against HSV-1. Type-specific immune responses to vaccine antigen may reveal differences in antibody activity against HSV-1 and HSV-2. Whether HSV-1 is more easily neutralized by vaccine-induced antibodies must be determined by further laboratory studies.

Preliminary data on correlates of protection against HSV-1 are available. Antibody, but not cellular immunity, was correlated with protection against HSV-1. Among the HSV vaccinees tested in the immunogenicity cohort, 8 were subsequently infected by HSV-1 and 10 by HSV-2. Subjects with HSV-1 infection had significantly lower gD-2 ELISA antibody titers at month 7 (mean titer, 3561) than subjects who remained uninfected (mean titer, $6875 ; \mathrm{P}=0.04$ ). This was not the case for subjects with HSV-2 infection (mean titer, 6339; $\mathrm{P}=0.78$ ). Cellular immune responses at month 2 or month 7 did not differ significantly between subjects who subsequently became infected with HSV-1 or HSV-2 and vaccinees who remained uninfected (see the Supplementary Appendix for details). 
The efficacy of the gD- 2 candidate vaccine against HSV-1 is important because epidemiologic studies suggest that sexual transmission of HSV-1 is increasing in the United States, ${ }^{1}$ although its prevalence among U.S. children is decreasing. ${ }^{11}$ Among control subjects in the present study, $60 \%$ of the cases of genital disease and two thirds of the infections (with or without disease) were caused by HSV-1 (Table 2). These data are similar to the finding that HSV-1 is the most common cause of genital herpes in college students ${ }^{12}$ and young heterosexual women, ${ }^{13}$ and similar trends are reported in other countries. ${ }^{14-18}$ HSV-1 now rivals HSV-2 as a cause of neonatal herpes disease. ${ }^{19}$ Public health officials and researchers will need to closely monitor sero-prevalence trends as future herpes vaccines are developed and assessed.

Although the development of a vaccine that provides protection against HSV-1 genital disease is a substantial step forward, additional progress is needed before a herpes vaccine is likely to be approved for general use. Any candidate vaccine will probably have to have proven efficacy against both HSV-1 and HSV-2 disease. Prevention of infection (with or without disease) is also an important goal, because asymptomatic genital shedding of HSV may lead to the spread of the virus to newborns or sexual partners. This is a more difficult goal to achieve and may require approaches such as live attenuated vaccines or vaccine vectors to generate protection.

\section{Supplementary Material}

Refer to Web version on PubMed Central for supplementary material.

\section{Acknowledgments}

Supported by a contract with the National Institute of Allergy and Infectious Diseases (N01-AI-45250) and by GlaxoSmithKline.

\section{References}

1. Xu F, Sternberg MR, Kottiri BJ, et al. Trends in herpes simplex virus type 1 and type 2 seroprevalence in the United States. JAMA. 2006; 296:964-73. [PubMed: 16926356]

2. Langenberg A, Benedetti J, Jenkins J, Ashley R, Winter C, Corey L. Development of clinically recognizable genital lesions among women previously identified as having "asymptomatic" herpes simplex virus type 2 infection. Ann Intern Med. 1989; 110:822-7.

3. Koutsky LA, Stevens CE, Holmes KK, et al. Underdiagnosis of genital herpes by current clinical and viral-isolation procedures. N Engl J Med. 1992; 326:1533-9. [PubMed: 1315930]

4. Stanberry LR, Spruance SL, Cunningham AL, et al. Glycoprotein-D-adjuvant vaccine to prevent genital herpes. N Engl J Med. 2002; 347:1652-61. [PubMed: 12444179]

5. Wald A, Zeh J, Selke S, et al. Reactivation of genital herpes simplex virus type 2 infection in asymptomatic seropositive persons. N Engl J Med. 2000; 342:844-50. [PubMed: 10727588]

6. Ashley R, Cent A, Maggs V, Nahmias A, Corey L. Inability of enzyme immunoassays to discriminate between infections with herpes simplex virus types 1 and 2. Ann Intern Med. 1991; 115:520-6. [PubMed: 1652909]

7. Bernstein DI, Aoki FY, Tyring SK, et al. Safety and immunogenicity of glyco-protein D-adjuvant genital herpes vaccine. Clin Infect Dis. 2005; 40:1271-81. [PubMed: 15825029]

8. Langenberg AGM, Burke RL, Adair SF, et al. A recombinant glycoprotein vac cine for herpes simplex virus type 2: safety and immunogenicity. Ann Intern Med. 1995; 122:889-98. [Erratum, Ann Intern Med 1995;123:395.]. [PubMed: 7755223]

9. Garland SM, Lee TN, Ashley RL, Corey L, Sacks SL. Automated microneutralization: method and comparison with Western blot for type-specific detection of herpes simplex antibodies in two pregnant populations. J Virol Methods. 1995; 55:285-94. [PubMed: 8609194] 
10. Margaret AS, Wald A, Huang ML, Selke S, Corey L. Optimizing PCR positivity criterion for detection of herpes simplex virus DNA on skin and mucosa. J Clin Microbiol. 2007; 45:1618-20. [PubMed: 17329447]

11. Xu F, Lee FK, Marrow RA, et al. Seroprevalence of herpes simplex virus type 1 in children in the United States. J Pediatr. 2007; 151:374-7. [PubMed: 17889072]

12. Roberts CM, Pfister FJ, Spear SJ. Increasing proportion of herpes simplex virus type 1 as a cause of genital herpes infection in college students. Sex Transm Dis. 2003; 30:797-800. [PubMed: 14520181]

13. Ryder N, Jin F, McNulty AM, Grulich AE, Donovan B. Increasing role of herpes simplex virus type 1 in first-episode anogenital herpes in heterosexual women and younger men who have sex with men, 1992-2006. Sex Transm Infect. 2009; 85:416-9. [PubMed: 19273479]

14. Vyse AJ, Gay NJ, Slomka MJ, et al. The burden of infection with HSV-1 and HSV-2 in England and Wales: implications for the changing epidemiology of genital herpes. Sex Transm Infect. 2000; 76:183-7. [PubMed: 10961195]

15. Buxbaum S, Geers M, Gross H, Schöfer H, Rabenau HF, Doerr HW. Epidemiology of herpes simplex virus types 1 and 2 in Germany: what has changed? Med Microbiol Immunol. 2003; 192:177-81. [PubMed: 12761673]

16. Suligoi B, Torri A, Grilli G, Tanzi E, Palú G. Seroprevalence and seroincidence of herpes simplex virus type 1 and herpes simplex virus type 2 infections in a cohort of adolescents in Italy. Sex Transm Dis. 2004; 31:608-10. [PubMed: 15388998]

17. Samra Z, Scherf E, Dan M. Herpes simplex virus type 1 is the prevailing cause of genital herpes in the Tel Aviv area, Israel. Sex Transm Dis. 2003; 30:794-6. [PubMed: 14520180]

18. Cunningham AL, Taylor R, Taylor J, Marks C, Shaw J, Mindel A. Prevalence of infection with herpes simplex virus types 1 and 2 in Australia: a nationwide population based survey. Sex Transm Infect. 2006; 82:164-8. [PubMed: 16581748]

19. Corey L, Wald A. Maternal and neonatal herpes simplex virus infections. N Engl J Med. 2009; 361:1376-85. [Erratum, N Engl J Med 2009;361:2681.]. [PubMed: 19797284] 


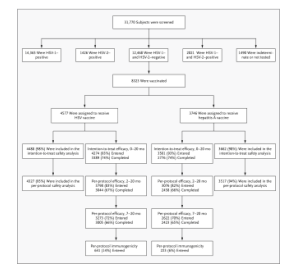

Figure 1. Randomization of Study Subjects

Both modified intention-to-treat and per-protocol analyses of efficacy were performed. Before the treatment assignments were revealed, an audit showed serious protocol violations at a single site; the sponsors declared that data from subjects at this site could not be evaluated for efficacy. The intention-to-treat analysis included all vaccinated subjects except those at the site with serious protocol violations, those who were found to be HSVseropositive at study entry, and those who did not return to the clinic after vaccination. The per-protocol analyses were further restricted to subjects who received either two doses of vaccine (per-protocol cohort for months 2 through 20) or three doses (per-protocol cohort for months 7 through 20) within protocol-specified windows, were uninfected at the start of each respective risk period, and did not have prespecified protocol deviations that might have confounded assessment of exposure, immunogenicity, or efficacy. Immunogenicity was evaluated in a random sample of subjects in the per-protocol cohort for months 7 through 20, with censoring of results after infection. A total of 31,770 women were screened by Western blot analysis for herpes simplex virus type 1 (HSV-1) and type 2 (HSV-2) antibodies for the Herpevac Trial, of whom 39\% were seronegative for both and $26 \%$ were vaccinated in the trial. The target treatment-assignment ratio was 1:1, but owing to a programming error, the initial subjects were randomly assigned at a 3:1 ratio in favor of the HSV-vaccine group. The data safety and monitoring board identified the problem, and randomization was corrected to a 1:1 ratio for the balance of the trial, resulting in a final ratio of 55:45 in favor of the HSV-vaccine group. 


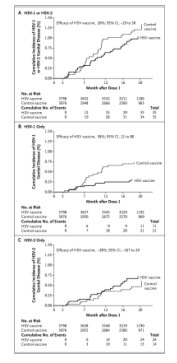

Figure 2. Cumulative Incidence of Genital Disease Caused by HSV in the Per-Protocol Cohort from Month 2 (1 Month after Dose 2 of Vaccine) through Month 20

Panel A shows the incidence of genital disease caused by either HSV-1 or HSV-2. Panel B shows the incidence caused by HSV-1 only. Panel C shows the incidence caused by HSV-2 only. 
Table 1

Demographic Characteristics and Risk Behaviors at Baseline in the Per-Protocol Cohort.

\begin{tabular}{|c|c|c|c|}
\hline \multirow[t]{2}{*}{ Variable } & $\begin{array}{l}\text { HSV-Vaccine Group }(\mathbf{N} \\
=\mathbf{3 7 9 8})\end{array}$ & $\begin{array}{c}\text { Control Group }(\mathrm{N}= \\
\text { 3076) }\end{array}$ & All Subjects $(N=6874)$ \\
\hline & \multicolumn{3}{|c|}{ number (percent) } \\
\hline \multicolumn{4}{|l|}{ Demographic characteristics } \\
\hline \multicolumn{4}{|l|}{ Age } \\
\hline $18-22 \mathrm{yr}$ & $2141(56)$ & $1703(55)$ & $3844(56)$ \\
\hline $23-26 \mathrm{yr}$ & $1115(29)$ & $974(32)$ & $2089(30)$ \\
\hline $27-30 \mathrm{yr}$ & $542(14)$ & $399(13)$ & $941(14)$ \\
\hline \multicolumn{4}{|l|}{ Race or ethnic group ${ }^{\dagger}$} \\
\hline White & $3040(80)$ & $2492(81)$ & $5532(80)$ \\
\hline Black & $389(10)$ & $301(10)$ & $690(10)$ \\
\hline Asian & $140(4)$ & $116(4)$ & $256(4)$ \\
\hline Other & $229(6)$ & $167(5)$ & $396(6)$ \\
\hline \multicolumn{4}{|l|}{ Risk behaviors ${ }^{t}$} \\
\hline Provided at least 1 response on risk-factor questionnaire & $3615(95)$ & $2934(95)$ & $6549(95)$ \\
\hline Reported ever having sex & $3245(85)$ & $2645(86)$ & $5890(86)$ \\
\hline Age $16-18$ yr when first had sex & $1749(46)$ & $1420(46)$ & $3169(46)$ \\
\hline More than 1 lifetime sexual partner & $2701(71)$ & $2231(73)$ & $4932(72)$ \\
\hline Reported using condoms & $2491(66)$ & $1959(64)$ & $4450(65)$ \\
\hline
\end{tabular}

* No significant differences were observed between treatment groups ( $\mathrm{P}>0.05$ for all comparisons). HSV denotes herpes simplex virus.

${ }^{\dagger}$ Race or ethnic group was determined by self-report. Other includes American Indian, Alaskan Native, Native Hawaiian, and Pacific Islander. Data on ethnic group are reported in the Supplementary Appendix.

"Risk-behavior questions were required only for subjects who responded "yes" to "ever had sex." The most frequently reported responses are presented. Detailed summaries of risk behaviors are provided in the Supplementary Appendix. 


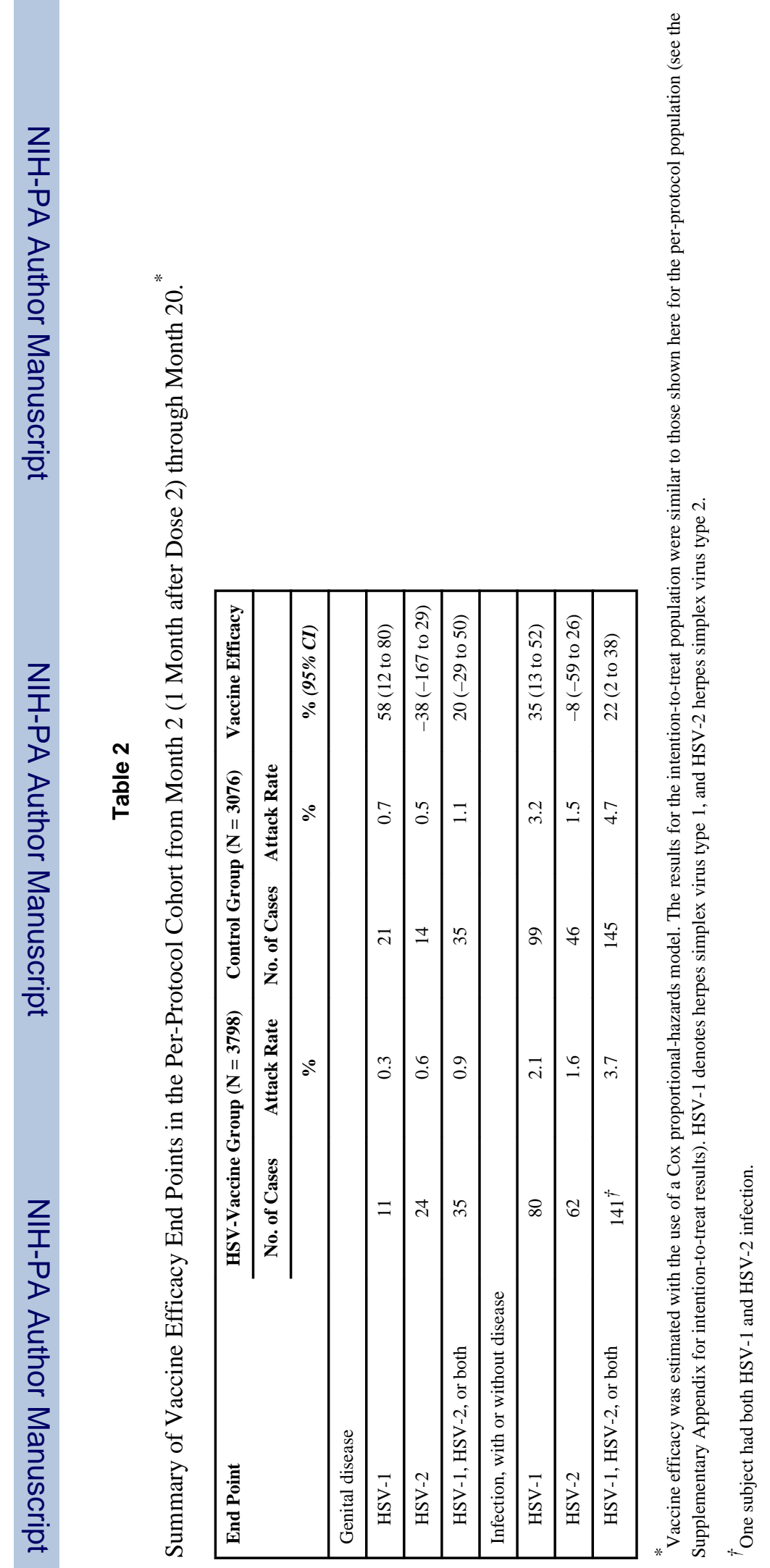

N Engl J Med. Author manuscript; available in PMC 2012 July 5. 
Table 3

Adverse Events in the Intention-to-Treat Safety Cohort.

\begin{tabular}{|c|c|c|c|}
\hline \multirow[t]{2}{*}{ Event } & $\begin{array}{l}\text { HSV-Vaccine Group } \\
(\mathbf{N}=\mathbf{4 4 8 8})\end{array}$ & $\begin{array}{c}\text { Control Group }(\mathbf{N}= \\
\text { 3662) }\end{array}$ & Odds Ratio (95\% CI) \\
\hline & \multicolumn{2}{|c|}{ no. $(\%)$} & \\
\hline \multicolumn{4}{|l|}{ Throughout the study } \\
\hline Death $^{\dagger}$ & $1(<1)$ & $0(0)$ & $\mathrm{NC}$ \\
\hline $\begin{array}{l}\text { Any serious adverse event, new-onset chronic disease, or } \\
\text { medically significant condition }\end{array}$ & $208(5)$ & $182(5)$ & $0.9(0.8-1.1)$ \\
\hline $\begin{array}{l}\text { Vaccine-related serious adverse event, new-onset chronic } \\
\text { disease, or medically significant condition } \S\end{array}$ & $10(<1)$ & $8(<1)$ & $\mathrm{NC}$ \\
\hline \multicolumn{4}{|l|}{ Within 31 days after administration of any dose ${ }^{I}$} \\
\hline Vaccine-related serious adverse event & $3(<1)$ & $3(<1)$ & $\mathrm{NC}$ \\
\hline Any unsolicited adverse event & $2154(48)$ & $1677(46)$ & $1.1(1.0-1.2)$ \\
\hline Grade 3 or higher unsolicited adverse event & $305(7)$ & $250(7)$ & $1.0(0.8-1.2)$ \\
\hline \multicolumn{4}{|l|}{ Within 7 days after administration of any dose } \\
\hline Any solicited injection-site event & $3937(88)$ & $2641(72)$ & $2.8(2.5-3.1)$ \\
\hline Redness & $1621(36)$ & $702(19)$ & $2.4(2.1-2.6)$ \\
\hline Swelling & $1371(31)$ & $438(12)$ & $3.2(2.9-3.6)$ \\
\hline Pain & $3902(87)$ & $2559(70)$ & $2.9(2.6-3.2)$ \\
\hline Any solicited systemic event & $2731(61)$ & $2071(57)$ & $1.2(1.1-1.3)$ \\
\hline Fatigue & $2031(45)$ & $1503(41)$ & $1.2(1.1-1.3)$ \\
\hline Fever & $400(9)$ & $260(7)$ & $1.3(1.1-1.5)$ \\
\hline Headache & $1885(42)$ & $1456(40)$ & $1.1(1.0-1.2)$ \\
\hline Malaise & $1459(33)$ & $1003(27)$ & $1.3(1.2-1.4)$ \\
\hline
\end{tabular}

* The intention-to-treat safety cohort excludes 171 subjects at a single site (which was unable to complete study monitoring as planned owing to a natural disaster) and 2 subjects without any follow-up data after vaccination. NC denotes not calculated.

${ }^{\dagger}$ The only death that occurred (in the HSV-vaccine group) was unrelated to vaccination.

New-onset chronic diseases and medically significant conditions were assessed by medical monitors at GlaxoSmithKline and the National Institutes of Health who were unaware of study assignments.

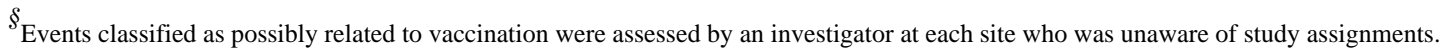

${ }^{\text {II }}$ Because of a programming error, 148 subjects received the incorrect product and were offered a fourth dose to complete their assigned vaccination sequence. A total of 112 subjects in the intention-to-treat safety cohort received a fourth dose, and safety data related to dose 4 are included in this analysis. 